\title{
A Radio Frequency Identification System for accurate indoor localization
}

\author{
Akshay Athalye, Vladimir Savic, Miodrag Bolic and Petar M. Djuric
}

\section{Linköping University Post Print}

N.B.: When citing this work, cite the original article.

(C2011 IEEE. Personal use of this material is permitted. However, permission to reprint/republish this material for advertising or promotional purposes or for creating new collective works for resale or redistribution to servers or lists, or to reuse any copyrighted component of this work in other works must be obtained from the IEEE.

Akshay Athalye, Vladimir Savic, Miodrag Bolic and Petar M. Djuric, A Radio Frequency Identification System for accurate indoor localization, 2011, Proc. of IEEE Intl. Conf. on Acoustics, Speech and Signal Processing (ICASSP), 1777-1780.

http://dx.doi.org/10.1109/ICASSP.2011.5946847

Postprint available at: Linköping University Electronic Press

http://urn.kb.se/resolve?urn=urn:nbn:se:liu:diva-81421 


\title{
A RADIO FREQUENCY IDENTIFICATION SYSTEM FOR ACCURATE INDOOR LOCALIZATION
}

\author{
Akshay Athalye, ${ }^{*}$ Vladimir Savić, ${ }^{\dagger}$ Miodrag Bolić,,${ }^{\ddagger}$ and Petar M. Djurić ${ }^{* *}$ \\ * Center of Excellence of Wireless and Information Technology, Stony Brook University, USA, e-mail: akshay.athalye@ cewit.stonybrook.edu \\ ${ }^{\dagger}$ Signal Processing Applications Group, Universidad Politecnica de Madrid, Spain, e-mail: vladimir@gaps.ssr.upm.es \\ ¥ School of Information Technology and Engineering University of Ottawa, Canada, e-mail: mbolic@ site.uottawa.ca \\ ** Department of Electrical \& Computer Engineering, Stony Brook University, USA, e-mail:djuric@ece.sunysb.edu
}

\begin{abstract}
In this paper we present a novel Radio Frequency Identification (RFID) system for accurate indoor localization. The system is composed of a standard Ultra High Frequency (UHF), ISO-18006C compliant RFID reader, a large set of standard passive RFID tags whose locations are known, and a newly developed tag-like RFID component that is attached to the items that need to be localized. The new semi-passive component, referred to as sensatag (sense-a-tag), has a dual functionality wherein it can sense the communication between the reader and standard tags which are in its proximity, and also communicate with the reader like standard tags using backscatter modulation. Based on the information conveyed by the sensatags to the reader, localization algorithms based on binary sensor principles can be developed. We present results from real measurements that show the accuracy of the proposed system.
\end{abstract}

Index Terms - indoor localization, RFID, passive tags

\section{INTRODUCTION}

Radio Frequency IDentifications (RFID) is a well-known technology for real-time identification of various assets and users. One of the main goals of RFID technology is to enable ubiquotous asset visibility. Accurately determining the location of an asset is of great importance in achieving this goal. Accurate localization using RFID can enable several applications such as location of tagged items in warehouses, and location of assets and personnel in hospitals and offices [5]. State-of-the-art localization methods can be broadly classified into three categories [2]: i) distance-based methods, ii) sceneanalysis, and iii) proximity-based methods. Distance-based methods rely on range measurements that can be based on Received Signal Strength (RSS), Time Of Arrival (TOA), or Time Difference of Arrival (TDOA). Using such measurements at (at least three) different reference points and upon converting them to estimated distances, one can employ simple trilateration to achieve localization. Scene-analysis methods consist of two phases. First, environmental

This work was supported by the NSF SBIR grant IIP-0912774. V. S. was supported by the FPU fellowship from the Spanish Ministry of Science and Innovation. We also thank partial support by ICT, project FP7-ICT-2009-4-248894-WHERE-2, the Spanish National Project under Grants TEC2009-14219-C03-01 and TEC2010-21217-C02-02-CR4HFDVL, and CONSOLIDER-INGENIO 2010 under grant CSD2008-00010 COMONSENS. P. M. D. was supported by NSF under Award CCF-1018323 and by the ONR under Award N00014-09-1-1154. We also thank the support of the Center of Excellence of Wireless and Information Technology at Stony Brook University. information (fingerprints) is acquired. Then, the target location is estimated by matching the measurements with the stored fingerprints, i.e., the estimated position is the average of the $k$ closest matches.

These methods are affected by dynamic changes in the environment. One direction of investigation for resolving this problem is to work with proximity-based methods which use binary information, i.e., information about a target being within the ranges of the reference tags or not. The location estimate is found either by associating the location of the target with that of the closest reference tag, or as the centroid obtained from the locations of all the reference tags that detected the target. For full details about RFID localization methods and quantitative comparisons, we refer the reader to $[2,5,7]$.

In this paper, we introduce a novel type of semi-passive Ultra High Frequency (UHF) RFID tag that has the capability to detect and decode backscatter signals from RFID tags in its proximity and to communicate this information to a standard RFID reader [3]. We refer to this tag as sensatag (from sense-a-tag). We show that when a sensatag is attached to an object that needs to be localized and the object is in an indoor environment that is populated with passive tags with known locations, one can estimate the location of the object with high accuracy.

The paper is organized as follows. In Section 2, we introduce the sensatags and in Section 3, we present the RFID system used for localization and the processing of data collected from this system. The experimental results of our work that demonstrate the performance of the system are shown in Section 4. We conclude the paper with some final remarks in Section 5.

\section{A NOVEL TYPE OF RFID COMPONENT}

Passive and semi-passive UHF RFID tags do not have on-board radios. They communicate with the reader using the principle of backscatter modulation wherein, the reflection cross section (RCS) of the tag antenna is varied in accordance with the data to be conveyed to the reader [6]. This modulates the signal reflected from the tag antenna to the reader. This tag backscatter is a weak signal that is further affected by multipath reflections and other ambient interferences in cluttered indoor environments like warehouses, retail stores, libraries, and offices [1]. This results in a low signal to noise ratio (SNR) for the tag response received by the reader. Hence conventional location techniques based on the measurement of some characteristic of the tag's response like RSS, TOA, or TDOA become highly inaccurate and unreliable for localization with passive and semi-passive RFID systems.

Our approach to localization is based on the addition of a new component to a standard reader-tag RFID system, called sensatag 


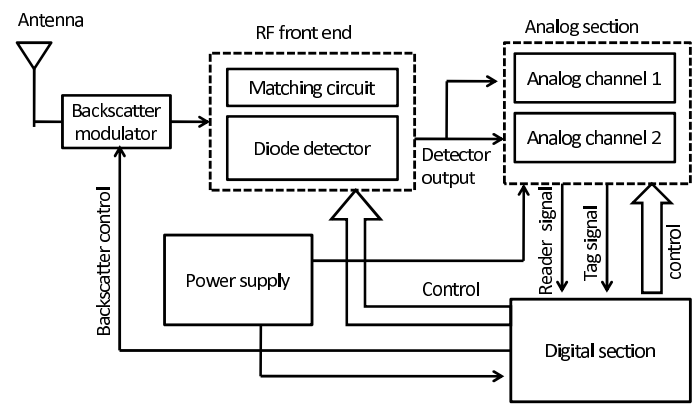

Fig. 1: Block diagram of the sensatag.

[3]. This semi-passive, tag-like component has the following capabilities: i) to detect and decode backscatter signals from RFID tags in its proximity and ii) to communicate with the reader using backscatter modulation.

On top of these basic capabilities, we have incorporated into the sensatag, a novel locator protocol, which is fully compatible with the EPC Global Class 1 Gen 2 standard (ISO-18006C). This protocol enables the sensatag to communicate with a standard reader and convey binary information about the presence or absence of a responding tag in its proximity.

A block diagram of the sensatag hardware is shown in Figure 1. The sensatag communicates passively without an on board radio. An on board battery is used for powering up the sensatag circuitry. Thus, in its current form, the sensatag is a semi-passive device. We will now briefly describe the various functional blocks that make up the sensatag.

\subsection{RF Front End}

The RF front end of the device consists of a passive envelope detector that is built using a Schottky Diode with corresponding matching circuit. When a passive RFID tag in the vicinity of the sensatag backscatters, the sensatag receives a signal that is a superposition of the tag backscatter and the continuous wave (CW) signal that the reader is transmitting during this time. The sensitivity of the sensatag to tags in its vicinity depends upon its ability to detect small changes in resultant power in this superimposed signal. This corresponds to the $\triangle$ RCS of the tag, i.e. the difference in tag antenna RCS when the tag backscatters a 1 vs when it backscatters a 0 . This means that the detector circuit needs to be optimized not for the maximum value of output voltage for a stated input power, but for maximum slope of the input power $\left(P_{i n}\right)$ vs output voltage $\left(V_{\text {out }}\right)$ characteristic around the typical power levels of operation. This optimization was done by appropriately tuning the matching circuit and the time constant of load on the baseband side of the diode detector circuit. The signal at the output of the diode detector is shown in Figure 2.

\subsection{Analog Section}

The sensatag analog section has the ability to process both the reader signal as well as the tag backscatter in order to produce a digital signal that can be processed by the digital section.

The analog processing of the reader signal is exactly the same as in a standard passive tag. It consists of a buffer followed by a hys-

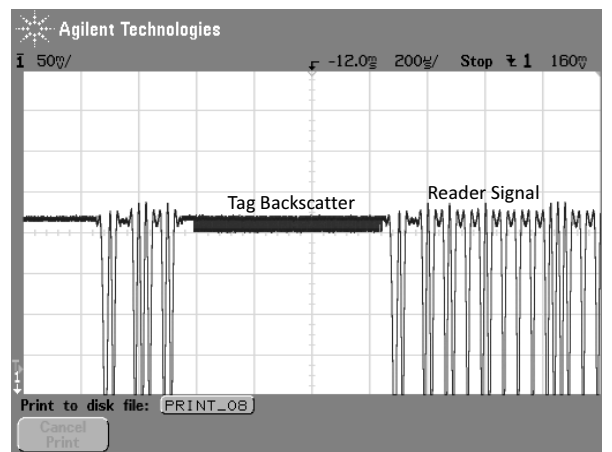

Fig. 2: Waveform of the signal captured by the sensatag.

teresis comparator that generates the digital output. The processing of the tag backscatter is a bit more complex since the backscatter is a weak signal that has a significant DC offset due to the presence of the $\mathrm{CW}$ signal from the reader. The circuit consists of a band-pass filter (or a high-pass filter) for removing the DC offset, followed by a comparator that is configured as a data slicer. The filter parameters and the threshold generation circuit for the comparator are adaptive.

\subsection{Digital Section Implementation:}

The digital section runs the sensatag protocol and as such is the brain of the device. In the current version, the digital section is implemented on an FPGA platform. This platform is chosen to allow for rapid prototyping and verification of the digital section, particularly keeping in mind that ultimately, the sensatag will be implemented as an ASIC. The present embodiment uses a Xilinx Spar$\tan$ 3AN FPGA. This device has an internal configuration memory which results in significant space saving on the digital section of the board. The current embodiment of the sensatag, used in the system described herein, is shown in Figure 3.

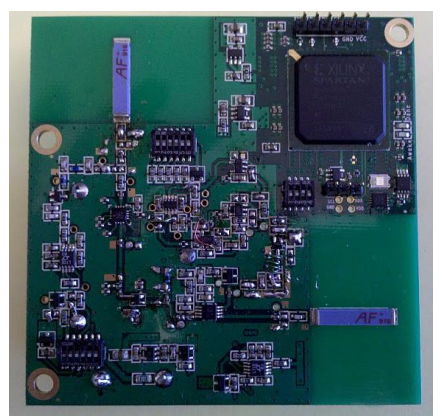

Fig. 3: Picture of the Sensatag board used in the experiments

\subsection{Locator Protocol}

As mentioned earlier, the sensatag implements a novel locator proto$\mathrm{col}$ which enables it to convey binary association information about tags in its vicinity to a standard reader. In order to implement this functionality, the locator protocol specifies two states of operation for the sensatag. In the first state or the listen state, the sensatag listens for backscattering tags in its vicinity. In the second state or the 
respond state, the sensatag itself functions as an RFID tag and conveys the information of the tags detected when it was in the listen state as part of its EPC ID payload. The transition between the two states is done based on different types of queries $\left(Q_{t}\right.$ - tag query and $Q_{s}$ sensatag query) received from the reader using the Select functionality provided by the Gen 2 standard. In the query round $Q_{t}$ the sensatag acts as a sensor detecting, decoding and storing information about the responding tags within its vicinity. In the subsequent $Q_{s}$ query round, the sensatag conveys the binary tag association, along with its own unique identifier information to the reader using backscatter modulation. The localization algorithm running on the reader side aggregates the binary association information from successive query rounds and determines the location of the sensatag with respect to the pre-deployed tags in the environment. The localization algorithms are described in detail in the next section.

\section{SYSTEM DESCRIPTION AND DATA PROCESSING}

In the system described in this paper, passive RFID tags are deployed at pre-defined locations within the environment where localization is to be performed. A sensatag is attached to the target of interest. The reader is programmed to send out alternating queries for the tags and sensatags using the Select functionality. The sensatag attached to the target operates using the locator protocol described above and conveys binary information about presence or absence of responding tags to the reader.

Let us assume that we have $M$ reference (passive) tags with known two-dimensional positions, $x_{i}(i=1,2, \cdots, M)$ and one sensatag with unknown position $l$. A reference tag can be detected by a sensatag with probability $p_{i}$. This probability depends on various factors, but primarily on the distance between the reference tag and the sensatag, orientation, and the power of the reader. This probability is easily estimated by counting the number of detections of a tag by a sensatag in a fixed number of reader queries.

Our main goal is to develop an algorithm that can perform well in environments with dynamical changes, and therefore we decided to use three simple localization methods that should work well in such circumstances. They are based on i) association, ii) centroids, and iii) weighted centroids.

With association we simply associate the sensatag with the nearest passive tag. The proximity is measured by comparing the $p_{i} \mathrm{~s}$ of each reference tag. The main drawback of association is when more passive tags are detected by the sensatag, the $p_{i}$ s may not correctly reflect the distance from the sensatag, which will imply that the sensatag will be associated with a wrong passive tag. As a result, the position error will be larger.

One simple way of building a more robust method is to implement averaging of the positions of all the passive tags that have been detected by the sensatag. In that case, the position of the sensatag is computed by: $\hat{l}=\sum_{i} x_{i} / N$, where the summation is over the locations of the tags that have been detected and $N$ is the total number of detected tags by the sensatags. Therefore, the estimated position will be the centroid of the positions of the detected passive tags. This approach does not take into account the number of detections.

A natural extension of the centroid method, is the weighted centroid, where the estimated position is the weighted average of the positions of the detected tags. Since it is expected that the closer tags will be detected more times than more distant ones, the weights are proportional to probabilities of detection of the tags. So, the estimated position is found as $\widehat{l}=\sum_{i} p_{i} x_{i}$. A similar idea is already used for target tracking in binary sensor networks [4].

\section{EXPERIMENTAL RESULTS}

Here we provide details of the experiments for studying our system and the performance of the methods for localization. We deployed 12 passive tags in 6 reference points, where at each point we deployed two passive tags. ${ }^{1}$ The overall area was $1.6 \mathrm{~m} \times 1.3 \mathrm{~m}$. The difference between the reader antenna and the center of the plane in which the sensatag is placed is $1.8 \mathrm{~m}$. The sensatag was placed somewhere inside the area of interest. The objective was to estimate its position in the area.

In the first set of experiments, we studied the accuracy of the estimate as a function of the reader power. We carried out localization of the sensatag at 10 different positions and computed the average error (defined as the Euclidean distance between the true and the estimated position) as a function of power. The results are shown in Figure 4, where we see that the association method has the worst performance but is almost constant in the studied range of reader powers. The method based on the weighted centroid outperformed the one that uses the centroid. For both methods the performance improved with increasing of the reader power. The best performance of all the methods was by the weighted centroid with a reader power of $28 \mathrm{dBm}$ (the accuracy was about $14 \mathrm{~cm}$ ).

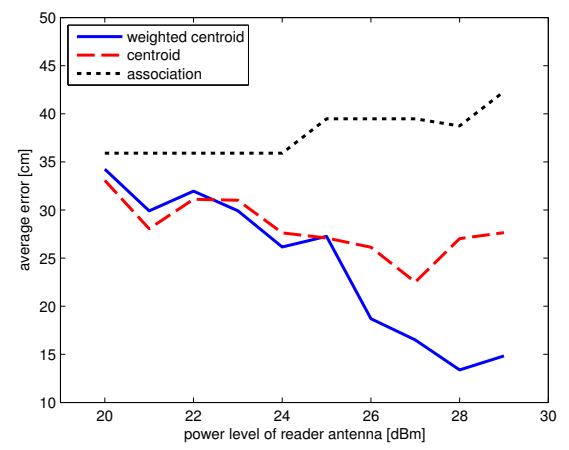

Fig. 4: The effect of reader power on the average position error of the three methods.

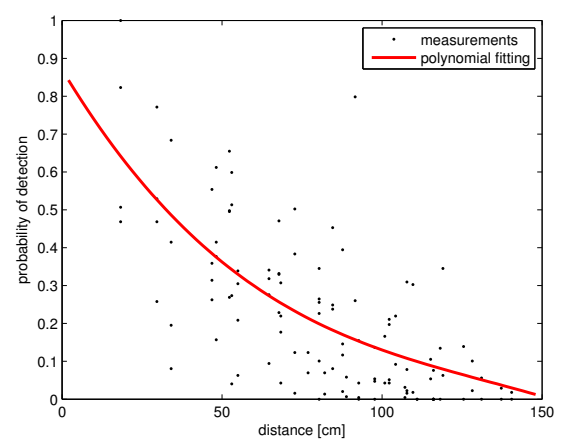

Fig. 5: Estimated probability of detection and the corresponding four-degree polynomial fitting.

In the next experiment, we studied the effect of the distance between the sensatag and the passive tags on the probability of detection. To that end, we acquired 20 independent measurements at 20

\footnotetext{
${ }^{1}$ The reason for having two passive tags at the same location was to prevent missing a tag by the sensatag because of destructive superposition of the signals from the reader and the tag.
} 


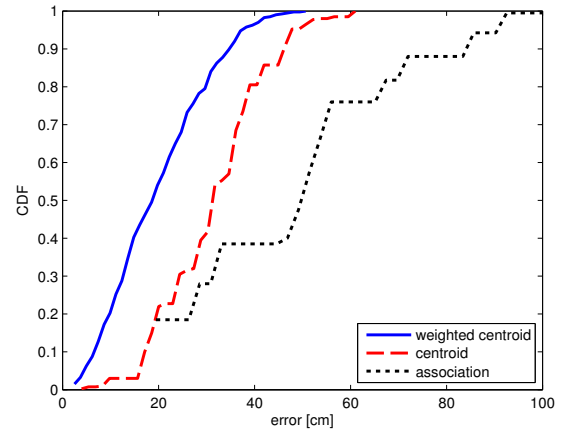

Fig. 6: Cumulative distribution function (CDF) of position error.

grid points. The results are shown in Figure 5. We can see that the probability of detection can vary considerably even for the same distance. We, however, expect this variability; it is due to the different multipath components and other factors that play role in formation of the signal received by the sensatag. We fitted the data with fourdegree polynomial function, which is also shown in the figure. The curve shows how the probability of detection decreases monotonically with distance.

The decrease of the probability of detection with distance is the main motivation for using the weighted centroid method. Clearly, with weighting the locations of the detected passive tags, we give higher emphasis to the detected tags that are closer than the ones that are further away from the sensatag.

For comparison of the performance of the methods, we also used the empirical cumulative distribution function (CDF) of the location error of the three methods. The results are shown in Figure 6. The CDFs of the errors confirm that the weighted centroid performs significantly better than the other two methods. For example, the probability of the error being less than $40 \mathrm{~cm}$ is about 0.95 for the weighted centroid, 0.82 for the centroid, and 0.4 for the associationbased method.

Finally, we also show the average error and the standard deviation for all the methods as functions of space (Figures 7-8). The weighted centroid-based method shows the smallest variability of the three methods across space. This is a feature that gives it an additional advantage over the other two methods.

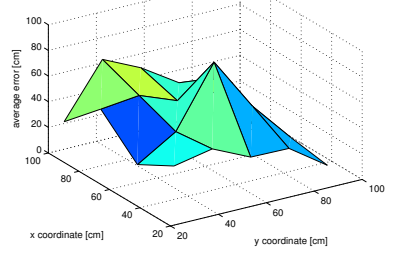

(a)

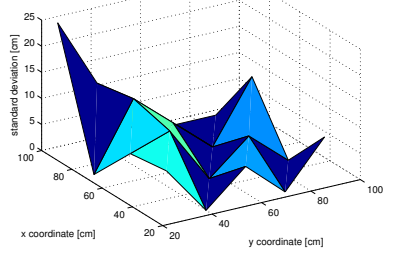

(b)
Fig. 7: Spatial distribution of (a) the average position error, and (b) the standard deviation for the association-based method.

\section{CONCLUSIONS}

In this paper we presented an UHF RFID system for accurate indoor localization of objects. The system is composed of (a) a num-

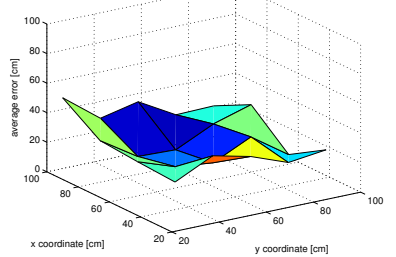

(a)

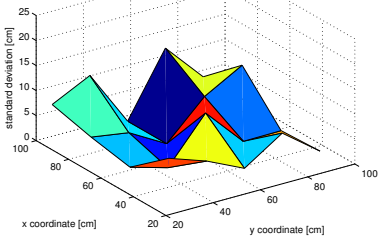

(b)
Fig. 8: Spatial distribution of (a) the average position error, and (b) the standard deviation for centroid-based method.

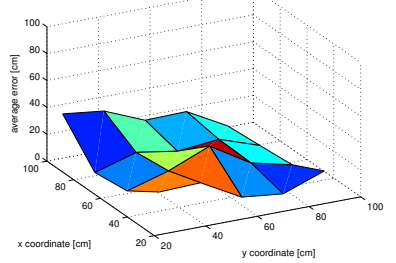

(a)

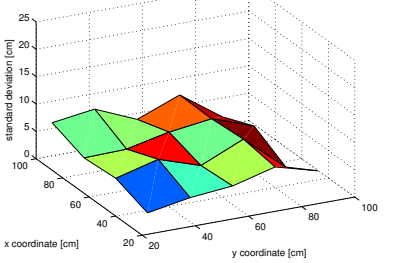

(b)
Fig. 9: Spatial distribution of (a) the average position error, and (b) the standard deviation for the weighted centroid-based method.

ber of passive tags whose locations are known and which serve as reference points, (b) a standard RFID reader, and (c) a new type of semi-passive tags called sensatags. Sensatags have the dual ability to (i) detect and decode backscatter signals from RFID tags that are in their proximity and (ii) communicate with the reader using backscatter modulation. When an object is tagged with a sensatag, it can be accurately located from the information acquired by the reader from the sensatag. We have conducted many experiments in which we studied the performance of our localization system. Some of the results are presented and show that the tagged object can be localized with high accuracy.

\section{REFERENCES}

[1] M. Bolic, A. Athalye, T. Li, "Performance of passive UHF RFID systems in practice," in M. Bolic, D. Simplot-Ryl, I. Stojmenovic, RFID Systems: Research trends and challenges, edited book, Wiley 2010.

[2] M. Bouet, and A.L. Dos Santos, "RFID tags: Positioning principles and localization techniques," in IEEE Proc. of IFIP Wireless Days, pp. 1-5, Nov. 2008.

[3] P. M. Djurić and A. Athalye, "RFID system and method for localizing and tracking a moving object with an RFID tag," Patent, Approved on: 2010-06-24; Application Number: 11799257, 2007.

[4] W. Kim, K. Mechitov, J.-Y. Choi, S. Ham, "On target tracking with binary proximity sensors," in IEEE/ACM Proc. of Information Processing in Sensor Networks (IPSN), pp. 301- 308, April 2005.

[5] T. Sanpechuda, L. Kovavisaruch, "A review of RFID localization: Applications and techniques," in IEEE Proc. of International Conference on ECTI-CON, pp.769-772, May 2008.

[6] D. M. Dobkin, The RF in RFID: Passive UHF RFID in Practice, Elsevier - Newnes, 2007.

[7] Y. Zhang, X. Li and M. Amin, "Principles and Techniques of RFID Positioning" in M. Bolic, D. Simplot-Ryl, I. Stojmenovic, RFID Systems: Research trends and challenges, edited book, Wiley 2010. 